\title{
Rab 25: Oncogenes and Tumor Suppressive of Cancer
}

\author{
Flora Chen ${ }^{1, *}$ \\ ${ }^{1}$ University of California, Davis, CA 95616, United States.
}

\begin{abstract}
Rab 25 is a small GTPase belonging to the RAS (rat sarcoma) superfamily. It is expressed in epithelial cells only and serves as a regulator of various intracellular signaling pathways. As a key player in in cell regulation, Rab 25 has been shown by research to function mainly as an oncogene in various cancers including breast cancer and ovarian cancer. However, Rab 25 has also been reported to be a tumor suppressor in cancer types such as colorectal cancer. A lot of research has been done about Rab 25 in recent years. This review is an overview of Rab 25, focusing on their role in human diseases such as cancer.
\end{abstract}

\section{Introduction}

The RAS (rat sarcoma) oncoprotein is a superfamily of small GTPases with more than 60 members identified in humans [1]. This superfamily can be subdivided into RAS, RHO, RAB, RAN and ARF [2]. The branch containing the most members is the Rab branch, a group of GTP-binding molecules with around 21-25 molecular $\mathrm{kDa}$ in weight that regulates intracellular protein transport [1]. Rab proteins act as transmitters, which induce the interaction between motor proteins and membranes, thus enabling transport vesicles to precisely bind to their targets [3]. A member of the Rab branch is the Rab 11 subfamily, which consists of Rab 11a, Rab 11b, and Rab 25 [4]. This subfamily coordinates apical transport and is only expressed in epithelial cells [5]. Among them, Rab 25 influences cellular physiology; and regulation of Rab 25 plays a critically functional role in treating diseases such as cancer. Regulation of Rab 25 represents an attractive new therapeutic avenue in molecular cancer therapeutics to precisely target neoplastic cells.

$\mathrm{Rab}$ proteins are master regulators of vesicular transport, providing a molecular identity to specialized cellular membrane compartments. Rab proteins are highly evolutionarily conserved with high sequence similarity. Rab proteins are constructed by a guanine nucleotide binding motif and a carboxyl terminus region. The sequence of their $\mathrm{C}$-terminus can be either $\mathrm{XXXCC}$, XXCCX, CCXX, CCXXX or XXCXC; and modification of such sequences allows the Rab protein to recognize and bind to specific vesicles [6]. The activity of Rab proteins is controlled by guanine nucleotides binding sites that attach to either GTP or GDP [7]. GDP-bound Rab proteins are inactive and located in the cytosol. Upon recognition by the guanine nucleotide exchange factor, GDP is replaced by GTP, making the Rab protein active [8]. Active Rab proteins then recruit various effector proteins to regulate vesicle sorting, uncoating, motility, tethering, and fusion [9]. After active Rab proteins finish coordinating the vesicle trafficking process, GTP is converted back into GDP by hydrolysis and the Rab protein becomes inactive once again [10].

\section{Structure of Signalling Function of Rab25}

Rab25 is a small GTPase with general GTPase tertiary structure formed by a six-stranded b-sheet barrel surrounded by a-helixs. Rab 25 serves as an activator of intracellular signaling pathways such as Akt, Wnt, and Src, and a suppressor of apoptotic pathways. When Rab 25 is overexpressed, it increases the phospho-Akt level, and may stimulate ovarian cancer cell proliferation [11]. When expression of Rab 25 is reduced, it lowers the cell migration capacity of cancer cell types including bladder cancer [12], hepatocellular carcinoma [13], and glioblastoma multiforme [14]. Knockdown of either Rab 25 or Akt inhibitor in ovarian cancer cells results in reduction of cisplatin resistance [15]. In addition, when Rab 25 is depleted, Wnt pathway target genes in hepatocellular carcinoma is inhibited [13]. The promoting effect of Rab 25 on cancer cells is regulated through increased expression of growth-stimulating pathways and suppression of apoptotic cell death pathways [7].

There are various mechanisms that influence the expression of Rab 25, such as the number of Rab 25 gene copies [7]. Studies have shown that in ovarian cancer, amplification of copy number upregulates Rab 25 expression [11]. Epigenetic regulation also alters Rab 25 expression [7]. When Rab 25 acts as an oncogene in ovarian cancer, it is negatively correlated with promoter methylation and RNA expression [16]. Reduction in Rab 25 methylation is linked to elevated levels of Rab 25 mRNA, indicating that epigenetic regulation can increase Rab 25 expression [16]. Studies also show that inhibition of DNA methylation can induce Rab 25 expression; and this induction is additionally in breast cancer cells when

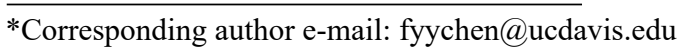


DNA methylation and histone deacetylases are coinhibited [17].

\section{Rab 25 And its Role in Cancer Progression}

As regulators of vesicle trafficking, Rab 25 has been identified by research to influence cancer cell development primarily as an oncogene [11]. Rab 25 promotes tumor progression through localizing integrinrecycling vesicles, which enhances the invasion capacity of tumor cells [7]. A report by Lapierre et al. indicates that in rat intestinal epithelial cells, overexpression of Rab 25 can transform cell shape from circular to spindle shaped through the internalization of $\alpha 5 \beta 1$-integrin [18]. The transformed cells formed tumors in mice that were immunocompromised [18]. Similarly, a study conducted by Jeong et al shows that through $\beta 1$ expression, Rab 25 can alter the morphology of mesenchymal phenotypes and lead to an increase in cell invasion and metastasis of cancer cells [19]. Furthermore, Rab 25 can strengthen the resistance of multiple types of cancer cells to drugs used in chemotherapy [20].

Furthermore, it was reported that knockdown of Rab25 can suppress the cell proliferation. Research shows that Rab 25 can promote the in vitro proliferation of renal cell carcinoma cells [21], hepatocellular carcinoma cells [13], and skin squamous cell carcinoma [22]. When levels of Rab 25 is reduced in lung cancer caused by tobacco carcinogen, tumor growth is reported to be restricted [23]. Conversely, ectopic expression of Rab 25 was reported to promote proliferation of breast cancer cells [24] and ovarian cancer cells [25].

Rab 25 was also reported to promote the in vitro migration and invasion capability of gastric cancer cells [26], breast carcinoma cells [27] and hepatocellular carcinoma cells [13]. In addition, knockdown of Rab 25 lowers in vitro cell migration rate as well as in vivo tumor metastasis in bladder cancer cells [12].

Cheng et al noted in their report that Rab 25 can delay in vivo occurrence of autophagy, a form of non-apoptotic cell death when the cell experiences nutrient deficiency, likely due to Rab 25's ability to induce endogenous amounts of ATP in ovarian cancer cells and maintain ATP levels after glucose and serum withdrawal [28]. Altogether, findings indicate that Rab 25 can promote aggressiveness of cancer by increasing the survival rate of cancer cells in acute nutrient depleted environments [28].

Moreover, Rab 25 can enhance resistance to chemotherapeutic agent cisplatin in tumor cells such as non-small cell lung cancer [29] and metastatic breast cancer [30]. A recent study demonstrates that Rab 25 can inhibit mitochondrial apoptosis in response to chemotherapy treatment in ovarian tumor as Rab 25 can directly regulate death priming [31]. When Rab 25 levels is reduced in environments containing low dose paclitaxel, etoposide or carboplatin, the survival rate of ovarian tumor cells decreased as a result. In addition, Rab 25 can maintain survival of ovarian cancer cells against serum starvation, anoikis and UV radiation [31].

\section{Rab 25 as A Suppressor of Tumor Cells}

Rab 25 can also act as a tumor suppressor in various types of cancer including esophageal squamous cell carcinoma [32]. colorectal adenocarcinomas [33], and head and neck squamous cell carcinoma [34]. In the report of Cheng et al., Rab 25 transduction inhibited the growth of epithelial human breast cancer cells and led to a reduction in the amount of viable tumor cells. In addition, Rab 25 expression increased tumor cell apoptosis and decreased migration capacity of tumor cells both in vitro and in vivo [35].

\section{The Dual Character of Rab 25}

According to recent studies, Rab25 can both enhance and suppress cancer progression. The dual character of Rab 25, as both an oncogene and a tumor suppressor, is likely cancer type- dependent. According to Leun's report, whether Rab 25 acts as an oncogene or a tumor suppressor is determined by the expressed levels of the Rab coupling protein (RCP). Increased expression of RCP could drive Rab 25's oncogenic functions [36]. A hypothesis by Dozynkiewicz et al. suggests that levels of chloride intracellular channel protein 3 (CLIC3) may be associated with the dual character of Rab 25. Dozynkiewicz et al. proposed that when CLIC3 is not present in the tumor cell, Rab 25 will terminate integrin signaling, likely causing Rab 25 to function as a tumor suppressor. Conversely, when CLIC3 is upregulated by Rab 25 in the tumor cell, integrins are not degraded and continue signaling, hence leading Rab 25 to function as an [37]. To better understand the association between CLIC3 level and the dual character of Rab 25, other major molecular components involved in the CLIC3-regulated recycling pathway need to be studied [37].

The oncogenic functions of Rab 25 is likely regulated by its promotion of the recycling of integrin to the plasma membrane and its stimulation of intracellular pathway signals [38]. Integrins such as $\alpha 5 \beta 1$ have been reported to be influential in the oncogenic functions of Rab 25 . According to Caswell et al., Rab25 enhances cell's invasive migration through $\mathrm{FN}$ rich matrices and through its association with integrin $\alpha 5 \beta 1$ [39]. Conversely, Nam et al reported that loss of $\beta 1$ integrin resulting from a deficiency of Rab 25 increased the formation of invasive colon tumors [33].

\section{Conclusion and Future Perspectives}

As the important regulators of crucial biological pathways and a key player in the maintenance of cellular functions, Rab 25 has received much attention for its function as either an oncogene or tumor suppressor. Increased expression of Rab 25 has reported to be positively correlated with tumor cell proliferation, migration, and invasion as well as resistance to autophagy and chemotherapeutic agents. Rab 25 's ability to recruit various effector proteins to partake in vesicle trafficking process as well as to activate intracellular signaling 
pathways such as Akt, Wnt, and Src prompts a successful regulation of the oncogenic functions of Rab 25.

The dual character of Rab 25 has not been studied thoroughly, though there are different hypotheses such as the expression of Rab coupling protein (RCP) drives the oncogenic functions of Rab 25 and the absence of CLIC 3 in tumor cells leads to Rab 25 functioning as a tumor suppressor. More research will need to be done to determine what mediates the dual functions of Rab 25 in cancer.

As Rab 25 is mainly an oncogene, it remains a good target for cancer therapeutic drugs. For example, the Rab25- $\alpha 5 \beta 1$ association presents a potential for this GTPase to be used as a biomarker. In addition, targeting the interaction between Rab25 and $\beta 1$ integrin can bring new treatment plans for cancer patients.

\section{References}

1. Pereira-Leal JB, Seabra MC. The mammalian Rab family of small GTPases: definition of family and subfamily sequence motifs suggests a mechanism for functional specificity in the Ras superfamily. J Mol Biol. 2000; 301:1077-87.W. Strunk Jr., E.B. White, The Elements of Style, third ed., Macmillan, New York, 1979.

2. Colicelli J. Human RAS superfamily proteins and related GTPases. Sci STKE. 2004; 250:RE13. [PubMed: 15367757]

3. Bhuin T, Roy JK. Rab11 in disease progression. Int J Mol Cell Med. 2015;4(1):1-8.

4. Goldenring JR, Shen KR, Vaughan HD, Modlin IM. Identification of a small GTP-binding protein, Rab25, expressed in the gastrointestinal mucosa, kidney, and lung. J Biol Chem. 1993 Sep 5;268(25):18419-22. PMID: 8360141.

5. JE, Wang X, Kumar R, Bhartur SG, Navarre J, Woodrum JE, Altschuler Y, Ray GS, Goldenring JR. Association of Rab25 and Rab11a with the apical recycling system of polarized Madin-Darby canine kidney cells. Mol Biol Cell. 1999; 10:47-61.

6. Fukuda M. Regulation of secretory vesicle traffic by Rab small GTPases. Cell Mol Life Sci. 2008; 65:2801-13.

7. Wang S, Hu C, Wu F, He S. Rab25 GTPase: Functional roles in cancer. Oncotarget. $2017 \mathrm{Jul}$ 26;8(38):64591-64599. doi: 10.18632/oncotarget.19571. PMID: 28969096; PMCID: PMC5610028.

8. Eathiraj S, Pan X, Ritacco C, Lambright DG. Structural basis of family-wide Rab GTPase recognition by rabenosyn-5. Nature. 2005; 436:4159.

9. Stenmark H. Rab GTPases as coordinators of vesicle traffic. Nat Rev Mol Cell Biol. 2009; 10:513-25.

10. Haas AK, Yoshimura S, Stephens DJ, Preisinger C, Fuchs E, Barr FA. Analysis of GTPase-activating proteins: Rab1 and Rab43 are key Rabs required to maintain a functional Golgi complex in human cells.
J Cell Sci. 2007; 120:2997-3010.

11. Cheng KW, Lahad JP, Kuo WL, Lapuk A, Yamada K, Auersperg N, Liu J, Smith-McCune K, Lu KH, Fishman D, Gray JW, Mills GB. The RAB25 small GTPase determines aggressiveness of ovarian and breast cancers. Nat Med. 2004; 10:1251-6.

12. Zhang J, Wei J, Lu J, Tong Z, Liao B, Yu B, Zheng F, Huang X, Chen Z, Fang Y, Li B, Chen W, Xie D, et al. Overexpression of Rab25 contributes to metastasis of bladder cancer through induction of epithelialmesenchymal transition and activation of Akt/GSK3ß/Snail signaling. Carcinogenesis. 2013; 34:2401-8.

13. Geng D, Zhao W, Feng Y, Liu J. Overexpression of Rab25 promotes hepatocellular carcinoma cell proliferation and invasion. Tumour Biol. 2016; 37:7713-8.

14. Ding B, Cui B, Gao M, Li Z, Xu C, Fan S, He W. Knockdown of Ras-Related Protein 25 (Rab25) Inhibits the In Vitro Cytotoxicity and In Vivo Antitumor Activity of Human Glioblastoma Multiforme Cells. Oncol Res. 2017; 25:331-40.

15. Fan Y, Wang L, Han X, Liu X, Ma H. Rab25 is responsible for phosphoinositide 3-kinase/AKT mediated cisplatin resistance in human epithelial ovarian cancer cells. Mol Med Rep. 2015; 11:2173-8.

16. Wrzeszczynski KO, Varadan V, Byrnes J, Lum E, Kamalakaran S, Levine DA, Dimitrova N, Zhang MQ, Lucito R. Identification of tumor suppressors and oncogenes from genomic and epigenetic features in ovarian cancer. PLoS One. 2011; 6:e28503.

17. Kikuchi M, Yamashita K, Waraya M, Minatani N, Ushiku H, Kojo K, Ema A, Kosaka Y, Katoh H, Sengoku N, Enomoto T, Tanino H, Sawanobori M, et al. Epigenetic regulation of ZEB1-RAB25/ESRP1 axis plays a critical role in phenylbutyrate treatmentresistant breast cancer. Oncotarget. 2016; 7:1741-53. http://doi.org/10.18632/ oncotarget.6480.

18. Lapierre LA, Caldwell CM, Higginbotham JN, Avant KM, Hall J, Beauchamp RD, Goldenring JR. Transformation of www.impactjournals.com/oncotarget 64599 Oncotarget rat intestinal epithelial cells by overexpression of Rab25 is microtubule dependent. Cytoskeleton (Hoboken). 2011; 68:97-111.

19. Jeong BY, Cho KH, Jeong KJ, et al. Rab25 augments cancer cell invasiveness through a $\beta 1$ integrin/EGFR/VEGF-A/Snail signaling axis and expression of fascin [published correction appears in Exp Mol Med. 2018 Sep 19;50(9):124]. Exp Mol Med. 2018;50(1):e435. Published 2018 Jan 26. doi:10.1038/emm.2017.248.

20. Mitra S, Cheng KW, Mills GB. Rab25 in cancer: a brief update. Biochem Soc Trans. 2012; 40:1404-8.

21. Li, Yuanyuan, et al. "Rab25 Upregulation Correlates with the Proliferation, Migration, and Invasion of Renal Cell Carcinoma." Biochemical and Biophysical Research Communications, vol. 458, no. 4, 2015, pp. 745-750., doi: 10.1016/j.bbrc.2015.01.144.

22. Jeong, Haengdueng, et al. "Loss of Rab25 Promotes 
the Development of Skin Squamous Cell Carcinoma through the Dysregulation of Integrin Trafficking." The Journal of Pathology, vol. 249, no. 2, 2019, pp. 227-240., doi:10.1002/path.5311.

23. Gankhuyag N, Yu KN, Davaadamdin O, Lee S, Cho WY, Park C, Jiang HL, Singh B, Chae CH, Cho MH, Cho CS. Suppression of Tobacco CarcinogenInduced Lung Tumorigenesis by Aerosol-Delivered Glycerol Propoxylate Triacrylate-Spermine Copolymer/Short Hairpin Rab25 RNA Complexes in Female A/J Mice. J Aerosol Med Pulm Drug Deliv. 2017; 30:81-90.

24. Zhang X, Lu Y, Shen G, Li J. Effect of RAB25 gene on proliferation of human breast cancer cell line MCF-7 in vivo and in vitro. Int J Clin Exp Pathol. 2016; 9:4746-55.

25. Gomez-Roman N, McGregor F, Wheate NJ, Plumb JA. Cucurbit [7] uril encapsulated cisplatin overcomes resistance to cisplatin induced by Rab25 overexpression in an intraperitoneal ovarian cancer model. J Ovarian Res. 2015; 8:62.

26. Cao C, Lu C, Xu J, Zhang J, Zhang J, Li M. Expression of Rab25 correlates with the invasion and metastasis of gastric cancer. Chin J Cancer Res. 2013;25(2):192-199. doi: 10.3978/j.issn.10009604.2013.03.01

27. Kessler, Daniel, et al. "The Action of Small GTPases Rab11 and Rab25 in Vesicle Trafficking During Cell Migration." Cellular Physiology and Biochemistry, vol. 29, no. 5-6, 2012, pp. 647-656., doi:10.1159/000295249.

28. Cheng KW, Agarwal R, Mitra S, et al. Rab25 increases cellular ATP and glycogen stores protecting cancer cells from bioenergetic stress. EMBO Mol Med. 2012;4(2):125-141 doi:10.1002/emmm.201100193

29. Ma, Y.f., et al. "Expression of Ras-Related Protein 25 Predicts Chemotherapy Resistance and Prognosis in Advanced Non-Small Cell Lung Cancer." Genetics and Molecular Research, vol. 14, no. 4, 2015, pp. 13998-14008., doi: 10.4238/2015.october.29.19.

30. Fan Y, Xu BH, Yuan P, Ma F, Wang JY, Ding XY, Zhang P, Li Q, Cai RG. Docetaxel-cisplatin might be superior to docetaxel-capecitabine in the first-line treatment of metastatic triple-negative breast cancer. Ann Oncol. 2013; 24:1219-25.

31. Temel, S.G., Giray, A., Karakas, B. et al. RAB25 confers resistance to chemotherapy by altering mitochondrial apoptosis signaling in ovarian cancer cells. Apoptosis (2020). https://doi.org/10.1007/s10495-020-01635-z.

32. Tong, M., et al. "Rab25 Is a Tumor Suppressor Gene with Antiangiogenic and Anti-Invasive Activities in Esophageal Squamous Cell Carcinoma." Cancer Research, vol. 72, no. 22, 2012, pp. 6024-6035., doi: 10.1158/0008-5472.can-12-1269.

33. Nam KT, Lee HJ, Smith JJ, Lapierre LA, Kamath VP, Chen X, Aronow BJ, Yeatman TJ, Bhartur SG, Calhoun BC, Condie B, Manley NR, Beauchamp RD, et al. Loss of Rab25 promotes the development of intestinal neoplasia in mice and is associated with human colorectal adenocarcinomas. J Clin Invest. 2010; 120:840-9.

34. Amornphimoltham, P., et al. "Rab25 Regulates Invasion and Metastasis in Head and Neck Cancer." Clinical Cancer Research, vol. 19, no. 6, 2013, pp. 1375-1388., doi: 10.1158/1078-0432.ccr-12-2858.

35. Cheng, J., Volk, L., Janaki, D. K., Vyakaranam, S., Ran, S., \&amp; Rao, K. A. (2010). Tumor suppressor function of Rab25 in triple-negative breast cancer. International Journal of Cancer. doi:10.1002/ijc. 24900

36. Luen Tang, B. Is Rab25 a tumor promoter or suppressor-context dependency on RCP status?. Tumor Biol. 31, 359-361 (2010). https://doi.org/10.1007/s13277-010-0030-z

37. Dozynkiewicz MA, Jamieson NB, Macpherson I, Grindlay J, van den Berghe PV, von Thun A, Morton JP, Gourley C, Timpson P, Nixon C, McKay CJ, Carter R, Strachan D, et al. Rab25 and CLIC3 collaborate to promote integrin recycling from late endosomes/lysosomes and drive cancer progression. Dev Cell. 2012; 22:131-45.

38. Agarwal R, Jurisica I, Mills GB, Cheng KW. The emerging role of the RAB25 small GTPase in cancer. Traffic. 2009; 10:1561-8.

39. Caswell PT, Spence HJ, Parsons M, White DP, Clark K, Cheng KW, Mills GB, Humphries MJ, Messent AJ, Anderson KI, McCaffrey MW, Ozanne BW. Rab25 associates with alpha5beta1 integrin to promote invasive migration in 3D microenvironments. Dev Cell. 2007; 13:496-510. 